\title{
Quarks and Their Discovery
}

\author{
Parashu Ram Poudel \\ Department of Physics, PN Campus, Pokhara \\ Email: parashupoudel@hotmail.com
}

\section{Introduction}

Quarks are the smallest building blocks of matter. They are the fundamental constituents of all the hadrons. They have fractional electronic charge. Quarks never exist alone in nature. They are always found in combination with other quarks or antiquark in larger particle of matter. By studying these larger particles, scientists have determined the properties of quarks. Protons and neutrons, the particles that make up the nuclei of the atoms consist of quarks. Without quarks there would be no atoms, and without atoms, matter would not exist as we know it. Quarks only form triplets called baryons such as proton and neutron or doublets called mesons such as Kaons and pi mesons. Quarks exist in six varieties: up (u), down (d), charm (c), strange (s), bottom (b), and top (t) known as quark flavors. Each quark has an antimatter charge (e) of one proton. The different flavors of quarks have different charges. The up (u), charm (c) and top ( $\mathrm{t}$ ) quarks have electric charge $+2 \mathrm{e} / 3$ and the down (d), strange (s) and bottom (b) quarks have charge -e/3; -e is the charge of an electron. The masses of these quarks vary greatly, and of the six, only the up and down quarks, which are by far the lightest, appear to play a direct role in normal matter.

There are four forces that act between the quarks. They are strong force, electromagnetic force, weak force and gravitational force. The quantum of strong force is gluon. Gluons bind quarks or quark and antiquark together to form hadrons. The electromagnetic force has photon as quantum that couples the quarks charge. The weak force causes the beta decay and allows a quark of one type to change

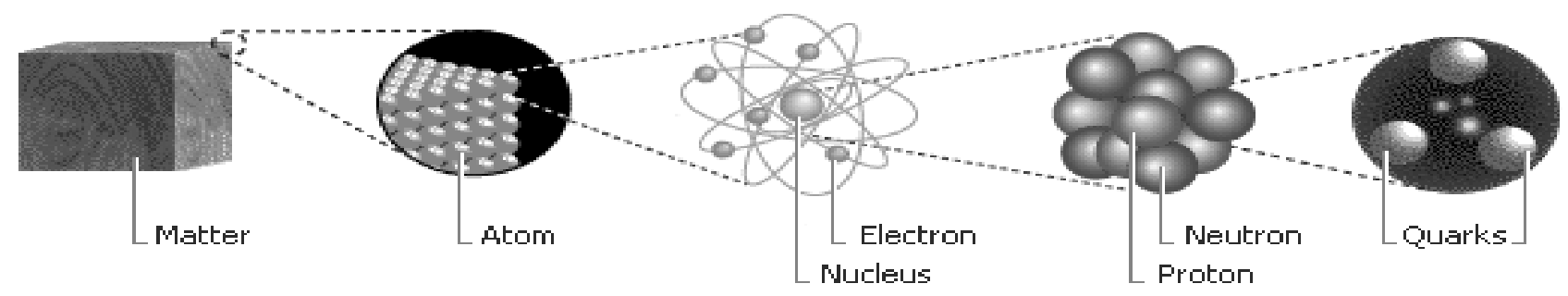

Fig-1 Quarks are building blocks of matter

counterpart called antiquark (designated by a line over the latter symbol) of opposite charge, baryon number, strangeness etc. The six flavors of quarks together with the six leptons (the electron, muon, tau, and their neutrinos) are truly elementary.

\section{Characteristics and Behaviour}

The six flavors of quarks are divided into three different categories called generations. The up and down quarks belong to the first generation, the charm and strange belong to the second generation, and the top and bottom belong to the third generation. Unlike other elementary particles, quarks have electric charge that is a fraction of standard charge i.e. the into another. The gravitational force couples quark mass. Table below shows quarks and their properties

Table -1 Quark and their properties

\begin{tabular}{|c|c|c|c|c|c|c|c|c|c|c|}
\hline $\begin{array}{l}\text { S. } \\
\text { N. }\end{array}$ & $\begin{array}{c}\text { Properties } \\
\text { Quarks }\end{array}$ & 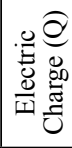 & $\begin{array}{c}\text { Spin } \\
(J)\end{array}$ & 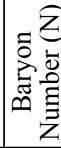 & 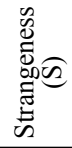 & 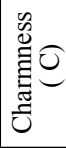 & 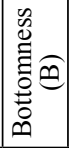 & 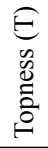 & 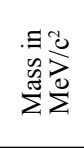 & $\begin{array}{c}\text { Anti- } \\
\text { quarks } \\
-\end{array}$ \\
\hline 1 & $\mathrm{Up}(\mathrm{u})$ & $+2 \mathrm{e} / 3$ & $1 / 2$ & $1 / 3$ & 0 & 0 & 0 & 0 & $3-9$ & $u$ \\
\hline 2 & Down (d) & $-e / 3$ & $1 / 2$ & $1 / 3$ & 0 & 0 & 0 & 0 & $1-5$ & $a$ \\
\hline 3 & Strange (s) & $-\mathrm{e} / 3$ & $1 / 2$ & $1 / 3$ & -1 & 0 & 0 & 0 & 125 & $S$ \\
\hline 4 & Charm (c) & $+2 \mathrm{e} / 3$ & $1 / 2$ & $1 / 3$ & 0 & +1 & 0 & 0 & 1,200 & c \\
\hline 5 & Bottom (b) & $-e / 3$ & $1 / 2$ & $1 / 3$ & 0 & 0 & -1 & 0 & 4,200 & $b$ \\
\hline 6 & Top (t) & $+2 \mathrm{e} / 3$ & $1 / 2$ & $1 / 3$ & 0 & 0 & 0 & +1 & 175000 & $t$ \\
\hline
\end{tabular}


Quarks Composition of Hadrons

Quarks bind together into states that can be observed directly in the laboratory as hadrons, particles with the strong force. The best-known examples of hadrons are the nucleons, the proton and neutron, from which all atomic nuclei are formed. The idea of quarks arose to explain the regularities of hadrons states, their charges and spins could be readily explained (and even predicted) by simply combining the then known $\mathrm{u}, \mathrm{d}$ and $\mathrm{s}$ quarks. The quark model consists of all these features. The confinement of quarks in hadrons is perhaps the most extraordinary feature of quarks A free quark, one that is separated from a nucleon, would be readily detectable because its charge would be $-2 / 3$ or $1 / 3$ of the charge of an electron. No convincing evidence for such a particle has been found, and it is now believed that in quarks confinement is an unavoidable consequence.

There are three quarks to make a proton or a neutron. The proton and neutron are different because the proton is a combination of two $u$ quarks and one $d$ quark and hence it has a total charge of $2(2 \mathrm{e} / 3)+$ $(-\mathrm{e} / 3)=+\mathrm{e}$. The neutron is made up of one $\mathrm{u}$ and two d quarks and hence it has total charge $(2 \mathrm{e} / 3)+$ $2(-\mathrm{e} / 3)=0$.Figure below shows quark composition of proton and neutron.

In addition to nucleons, other combinations of three quarks have been observed, and all are known collectively as baryons. For example, from the u, $\mathrm{d}$, and $\mathrm{s}$ quarks, it is possible to make ten distinct combinations, and all have been seen (notice that they all have charges that are integer multiples of e). In addition, baryons with $\mathrm{c}$ and $\mathrm{b}$ quarks have also been observed. All the baryons except for the proton and neutron decay quite rapidly because the weak interactions make all the quarks unstable.
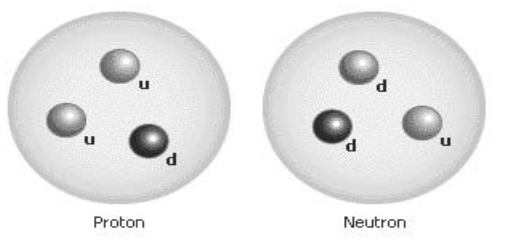

Baryons

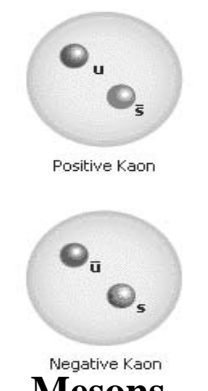

Mesons
Fig-2 Quarks in baryons and mesons
In addition to baryons, quarks can combine with their antiparticles, the antiquark, to form mesons. For example, the combinations $u s$ and $u \bar{s}$ can form the $\mathrm{K}^{-}$and $\mathrm{K}^{+}$, the Kaons, of electric charge-e and $+e$, respectively as shown in the figure. All the mesons are bosons with integer spins. The table below shows quark composition of some hadrons.

Table -3 Quarks Composition of Some Baryons And Mesons

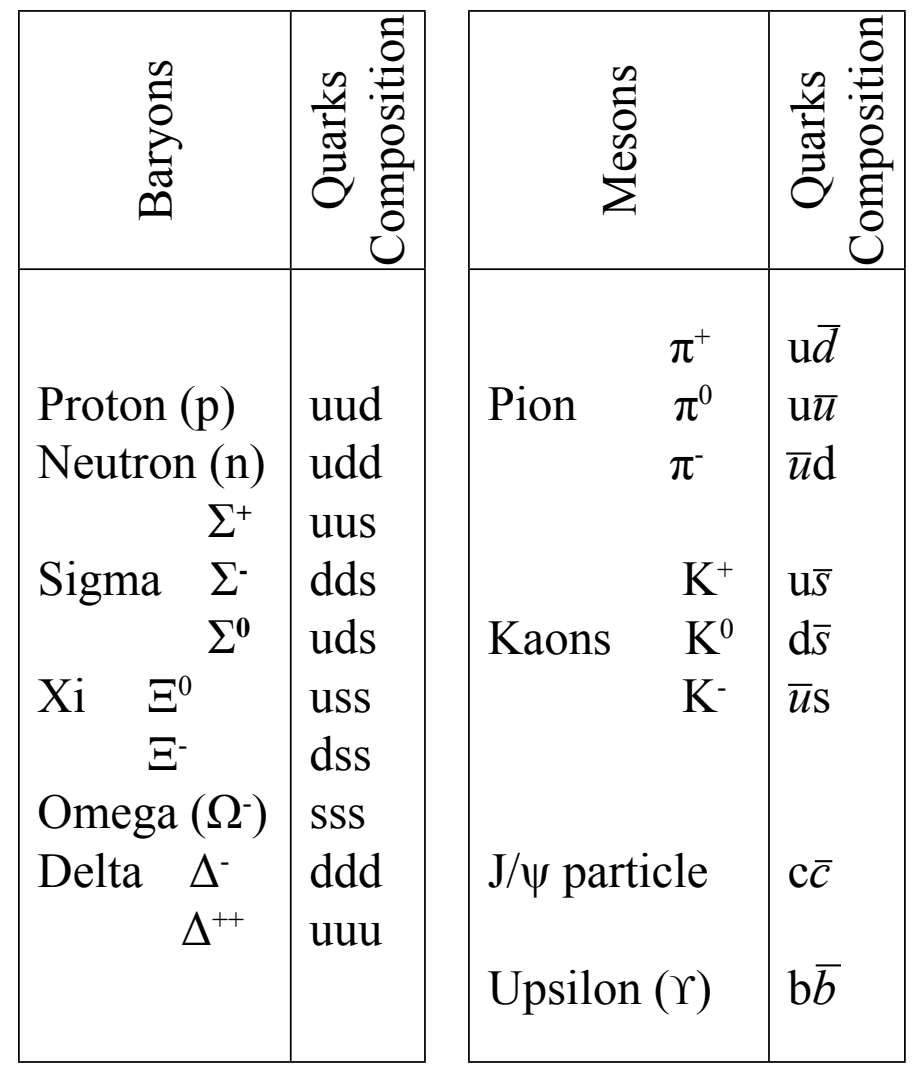

\section{Color of Quarks}

It is well known that particles are of two types: fermions, whose spins are odd multiples of $1 / 2$, and bosons, which have zero or integral spin. Fermions obey the Pauli's Exclusion Principle that states that two of them cannot occupy the same physical state simultaneously. The $\Omega^{-}$(sss) was found to have a spin of $3 / 2$, and the simplest configuration of the ground state to give this result would require all three quarks to have spins up. This violates the exclusion principle as the quarks are supposed to be fermions. This problem was resolved by the introduction of 
the concept of color, as formulated in quantum chromodynamics (QCD). In this theory of strong interactions color has nothing to do with the colors of the everyday world but rather represents a property of quarks that is the source of the strong force. The colors red, green, and blue are ascribed to quarks, and their opposites, antired, antigreen, and antiblue, are ascribed to antiquarks. According to QCD, all combinations of quarks must contain mixtures of these imaginary colors that cancel out one another, with the resulting particle having no net color. A baryon, for example, always consists of a combination of one red, one green, and one blue quark and so never violates the exclusion principle. Thus, the three quarks in the $\Omega^{-}$have their spins parallel and the same spatial wave function, but these are of three different colors. Since all the three colors are equally present, it is said that the state is color-neutral, and since this additional quantum number is not seen in real hadrons, one knows that these states must all be colorless. The baryons accomplish colorlessness by being composed of three different-colored quarks; the mesons are color-neutral as they are composed of quarks and antiquarks. All hadrons have zero net $\mathrm{r}, \mathrm{b}$ and $\mathrm{g}$ colors; they are all colorless.

The property of color in the strong force plays a role analogous to that of electric charge in the electromagnetic force, and just as charge implies the exchange of photons between charged particles, so does color involve the exchange of massless particles called gluons among quarks. Just as photons carry electromagnetic force, gluons transmit the forces that bind quarks together. Quarks change their color as they emit and absorb gluons, and the exchange of gluons maintains proper quark color distribution. The binding forces carried by the gluons tend to be weak when quarks are close together. Within a proton (or other hadrons), at distances of less than $10^{-15}$ meter, quarks behave as though they were nearly free. When one begins to draw the quarks apart, however, as when attempting to knock them out of a proton, the effect of the force grows stronger. This is because gluons have the ability to create other gluons as they move between quarks. Thus, if a quark starts to speed away from its companions after being struck by an accelerated particle, the gluons utilize energy that they draw from the quark's motion to produce more gluons. The larger the number of gluons exchanged among quarks, the stronger the effective binding forces become. Supplying additional energy to extract the quark only results in the conversion of that energy into new quarks and antiquarks with which the first quark combines. This phenomenon is observed at high-energy particle accelerators in the production of "jets" of new particles that can be associated with a single quark.

\section{Discovery of quarks}

Until the discovery of the electron by J.J. Thomson in 1897, it was assumed that atoms were the fundamental constituents of matter. This discovery, and Rutherford's discovery of atomic nucleus and the proton in 1911, made it apparent that atoms were not themselves elementary in the sense that they have an internal structure. Chadwick's discovery of the neutron in 1932 completed the atomic model based on an atomic nucleus consisting of protons and neutrons surrounded by sufficient electrons to balance the nuclear charge. It did not, however, explain the great stability of the nucleus, which clearly could not be held together by an electromagnetic interaction as the neutron has no electric charge. In 1935 Hideki Yukawa suggested that exchange forces that held them together involved short lived particles, called mesons, which jumped from protons to neutron and back again. This concept leads to the discovery of strong and weak interactions.

The first step in the discovery of quarks occurred in 1947 when a new particle, the K-meson, was discovered in cosmic rays. However, along with the discovery came a mystery. These particles were made in nuclear collisions by very-high-energy cosmic-ray particles via the strong force, with an interaction time of about $10^{-24} \mathrm{~s}$, which is approximated by dividing the radius of a nucleus by the speed of the cosmic-ray particle. The new particles were observed, strangely, to decay into pions with lifetimes of the order of $10^{-}$ ${ }^{9} \mathrm{~s}$. If the strong force could make these particles so easily, why did it not cause them to decay just as fast? They were named strange particles. Later, in 1953, these particles were made in the Cosmotron at 
Brookhaven National Laboratory (BNL), and soon the whole families of these particles were discovered. By 1961 two octets, one of baryons and the other of mesons and an additional decuplet of baryons had been discovered. In 1964 Murray Gell-Mann and George Zweig independently found the solution for explaining many of the observed properties of these particles. They proposed that all baryons were composed of triplets of quarks (selected from $\mathrm{u}, \mathrm{d}$ and s) and the mesons were doublets formed by a quark and an antiquark. Gell-mann borrowed the word quark from James Joyce's novel Finnegan Wake, which contains the phrase "three quarks for Muster Mark".

The development of the theory of quantum chromo dynamics (QCD), which describes how gluons interact among themselves and with the quarks, was started by the work of Yoichiro Nambu and by Oscar W. Greenberg in 1966. It was ultimately completed by 1973 and remains the underlying theory of the strong interaction.

Physicists continued to use the three quark model through the 1960s. Experiments conducted at the Stanford Linear Accelerator (SLAC) in Stanford, California during the early 1970s supported the model. In these experiments high energy electrons collided with a target of proton. These experiments were analogous to experiments performed by British Physicists Ernest Rutherford during the early 1900s. Rutherford determined the inner structure of atom by observing how particles scattered off atoms. In the 1970s scientists looked at how electrons scattered off protons to learn about the internal structure of proton. These experiments conclusively supported the existence of quarks and gluons inside protons. In 1990 a Nobel Prize in Physics was awarded to Henry W. Kendall, Jerome I. Friedman, and Richard E. Taylor for this discovery.

Even though physicists did not need more than three quarks to describe existing particles, American physicists Sheldon Glashow, Greek physicists John Iliopoulis and Italian physicists Luciano Maiani developed a theory in 1970 that predicted the existence of thefourth quark, called the Charm quark.
As early as in 1964, theorists had speculated on the existence of an additional quark. Ten years later at 1974, the Charm quark appeared simultaneously in experiments at SLAC and BNL, resulting in a Nobel Prize in Physics for Burton Richter and Samuel C. Ting. They discovered a meson, called the $\mathrm{J} / \psi$ particle, consisting of a charm and anticharm quark. The new quark was more massive than the proton at $1,200 \mathrm{MeV} / \mathrm{c}^{2}$ and had a charge of $2 \mathrm{e} / 3$ like the up quark. Soon experiments found new charmed mesons and baryons in which the charm quark formed states with the up and down quark; even charmed-strange states with the strange quark appeared.

In 1977 the tau lepton was discovered at SLAC by a collaboration led by Martin Perl, and the upsilon meson was discovered at Fermi lab by a collaboration led by Leon Lederman. The upsilon had a mass of about $9.5 \mathrm{GeV} / \mathrm{c}^{2}$ and it is composed of $\mathrm{a} b \bar{b}$ pair. States have also been discovered in which the bottom quark, with mass of about $4.5 \mathrm{GeV} / \mathrm{c}^{2}$ binds via the strong force with the other quarks to form baryons and mesons that are several times as massive as the proton. After the discovery of the tau lepton and bottom quark, there was much anticipation, based on the expectations mentioned above, that a sixth quark, the top, would exist. It was surprising that it took nearly twenty years before two groups at Fermi lab announced the discovery. The reason for the long delay was that the top has a mass of $175 \mathrm{GeV} / \mathrm{c}^{2}$. It was not until the Tevatron proton-antiproton collider came on-line that any machine had a high enough energy to make the top. It weighs about as much as a gold atom! Table-2 shows some of the important events in quarks history.

\section{Table-2: Some of the important milestone in quark history}

1935 Existence of mesons predicted.

1947 Discovery of the pie $(\pi)$ and first evidence for strange particles

1951 Discovery of strange mesons and strange baryons

1952 Hypothesis of associated production

1952 First evidence for strangeness 2 baryons

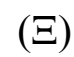


1953 Associated production confirmed at the Cosmotron

1961 Gell-Mann proposed Eight Fold Way for particle classification

1964 Gell-Mann and George Zweig proposed the quark model of sub-atomic structure

1964 Charm predicted

1964 Omega discovered at Brookhaven

1969 Electron-scattering experiments reveal substructure inside proton

1973 Formulation of Standard Model of forces

1974 Discovery of J/ $\psi$ at SLAC and BNL

1977 Discovery of bottom quark (upsilon meson) at FNAL

1979 Experimental observation of gluon jets

1995 Discovery of top quark at FNAL

On the basis of above findings and discussions it is understood that it took about half a century from the discovery of strangeness to the discovery of the top quark to complete what is called the Standard Model for quarks and leptons and their interactions. Physicists continue to conduct experiments to discover whether new data will dispute their findings and to learn more about the properties of quarks. Physicists do not know how long single quark exists (since they always found in other particle), or whether quarks could combine in any other ways. Scientists suspect that particles containing one lepton and one quark, called leptoquark, may exist but they have yet to find any. The questions of why there are only three families of quarks and leptons and an explanation of their masses remain to be answered. It has yet to be explained why the top quark is so much more massive than the other elementary particles Anyway, the twentieth century began with the conformation that the matter was not continuous but made of tiny atoms and molecules and ended with the conformation that matter is made, in part, of even tinier objects called quarks.

\section{Bibliography}

Alan Isaacs, Dictionary of Physics, 1996. Oxford University press, New York.

Arthur Beiser, Concept of Modern Physics, 1999. TMH New Delhi.

Feynmann, Leighton and Sands, Lectures on Physics, 2007. Narosa Publishing House, New Delhi.

John S. Rigden, Building Block of Matters, 2000. McGRAWHill, New York.

Kenneth S. Krana, Modern Physics, 2008. Wiley India Limited, New Delhi.

Poudel P.,Gautam A.,Adhikari B.,Thapa M.,Khatri M.,Principles of Physics, 2009. Ayam Publication, Kathmandu, Nepal.

R. Murugeshan, Modern Physics, 2009. S. Chand and Company, New Delhi.

Ray Spangenburg and Diane K. Moser, The History of Since from 1946 to the 1990, 1999. University Presss India Limited, Hydrabad.

W.E. Burcham and M. Jobes, Nuclear and Particle Physics, 1998. Addition Wesley England.

Young and Freedman, University Physics, 2009. Pearson Education Delhi.

Microsoft Corporation, Encarta, 2009. Microsoft Corporation.

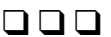

